\title{
An observation of age-related asymmetry in the complexity of verbal mediator components
}

\author{
PHILIP H. MARSHALL, JEFFREY W. ELIAS, and AVERY BRATT \\ Texas Tech University, Lubbock, Texas
}

\begin{abstract}
College-age and elderly subjects were required to give verbal mediators for consonant-vowelconsonant (CVC) pairs varying in associability level, where associability was defined as the scaled likelihood of a CVC pair's yielding a verbal mediator. The complexity of each elaboration of each of the components of the pairs was then determined to investigate potential differences in mediator formation between the two groups of subjects. In agreement with previous findings, the younger subjects tended to elaborate the stimulus term more than the response term. However, the elderly subjects elaborated the response term significantly more, and there was a trend for this to occur more with low-associability pairs.
\end{abstract}

Reviews of the use of verbal mediation by young and elderly subjects (Burke \& Light, 1981; M. F. Elias, P. K. Elias, \& J. W. Elias, 1977; Kausler, 1982) generally conclude that the elderly show less spontaneous use of verbal mediators and that when instructions make them generate mediators, they are still unlikely to perform on memory tasks at the same level as younger subjects.

In an earlier study, Marshall et al. (1978) considered the possibility that the inefficiency of mediator usage associated with increased age might be due partly to the fact that mediators generated by the elderly may somehow be structurally different from the mediators generated by younger subjects. In that study, however, it was found in assessing the quantitative aspects of mediator formation (complexity) and the qualitative aspects of mediator formation (types of mediators) that the young and the elderly exhibited no discernible differences. Marshall et al. used single consonant-vowel-consonant (CVC) items so that the analysis could follow the procedures established by Prytulak (1971) to analyze mediator complexity. However, it has been more common in studies of mediation to use the paired-associate procedure, in which the role of the mediator (which is to form a linkage between the components of the pair) may readily be observed. In a study by Owens, Werder, and Marshall (1974) on mediation, it was found that young subjects' mediators had significantly more embellishment for the stimulus members of CVC pairs. Those authors reasoned that their subjects had used an efficient strategy of keeping the response term relatively intact when generating the mediator, since upon later recall they would be better able to decode the mediator and retrieve the response term. Furthermore, this would be in line with Prytulak's (1971) conclusion that increasing complexity leads to increased difficulty in decoding the mediator to retrieve the original CVC term.

Requests for reprints should be sent to Philip H. Marshall, Department of Psychology, Texas Tech University, Lubbock, TX 79409.
The present study focused on the issue of mediator structure and aging by investigating the degree of embellishment of the component terms of mediators for CVC pairs. Complexity of the separate components of the pair was judged by the Prytulak (1971) procedure, which provides a count of the number of transformations of the nominal term used to generate the mediator. The present procedure encouraged subjects to generate two-word mediators to link the two CVC terms. This method allowed the complexity of the embellishment of the component terms to be analyzed. It was mentioned to the subjects that they might receive a recall test on these pairs, but in fact, no such test was administered. For the remainder of this discussion the letter A will signify the lefthand (traditionally the stimulus) component, and the letter $\mathrm{B}$ the right-hand (traditionally the response) component.

\section{METHOD}

\section{Subjects}

Eighteen college students (aged 18 to 22 years; mean, 19.5 years) and 18 elderly subjects (aged 61 to 82 years; mean, 67.5 years) volunteered to participate. All elderly subjects resided in private residences, reported themselves to be relatively healthy, and had at least a high school diploma. There were approximately equal numbers of men and women in both age groups.

\section{Materials \\ Forty-five CVC pairs, 15 for each of three levels of associability (high, medium, and low), from the norms of Montague and Kiess (1968) were used in this study. Associability is defined as the probability that a given CVC pair would yield a verbal natural language mediator (NLM) in a given time period. All $45 \mathrm{CVC}$ pairs were presented randomly in book- let form with one CVC pair per page, accompanied by a blank space for the subjects to write the NLM.}

\section{Procedure}

The subjects were run in small grups of 2 to 5 individuals. The procedures of paired-associate learning and the use of rote and mediation strategies were explained, and the efficiency of the mediation strategy was emphasized. Since the focus of this study was on the component analysis, it was stressed by example, but not made a requirement, that the 
NLM should be two words that would link the A and B components. Subjects were instructed to examine the pairs silently and then write down an appropriate NLM that they could use to facilitate their retention of the pair. Twenty-five seconds were allowed for each CVC pair. Although no recall of the material was in fact required, the instructions implied that the subjects would later be shown one member of the pair and asked to give the other. Marshall et al. (1978) have shown that actually giving the recall test does not change the structural properties of the NLMs.

\section{RESULTS}

Natural language mediators that met the criterion of having both $\mathrm{A}$ and $\mathrm{B}$ components available for the complexity analysis were determined for each subject. A given NLM met this requirement if separate elaborations were given for each component. Analysis of variance indicated that younger subjects produced more suitable (i.e., twoword) NLMs than the elderly subjects $(80 \%$ vs. $63 \%)$ $[F(1,34)=6.11, p<.05]$ and that the elderly subjects were significantly less likely to produce suitable NLMs for low-associability items $[F(2,68)=4.49, p<.05]$. Figure 1 presents these data.

Complexity scores were obtained for the two components of the acceptable NLMs by applying Prytulak's (1971) procedure, which is a count of the number of transformations made in changing a nominal CVC item into an NLM (see Marshall et al., 1978). An analysis of variance with age as the between-subjects factor and associability level as the within-subjects factor found a significant main effect for associability $[F(2,68)=124.37$, $p<.001$ ], with the highest complexity scores appearing for pairs low in associability (mean $=2.36, S D=$ .42 ) which differed significantly (Newman-Keuls) from

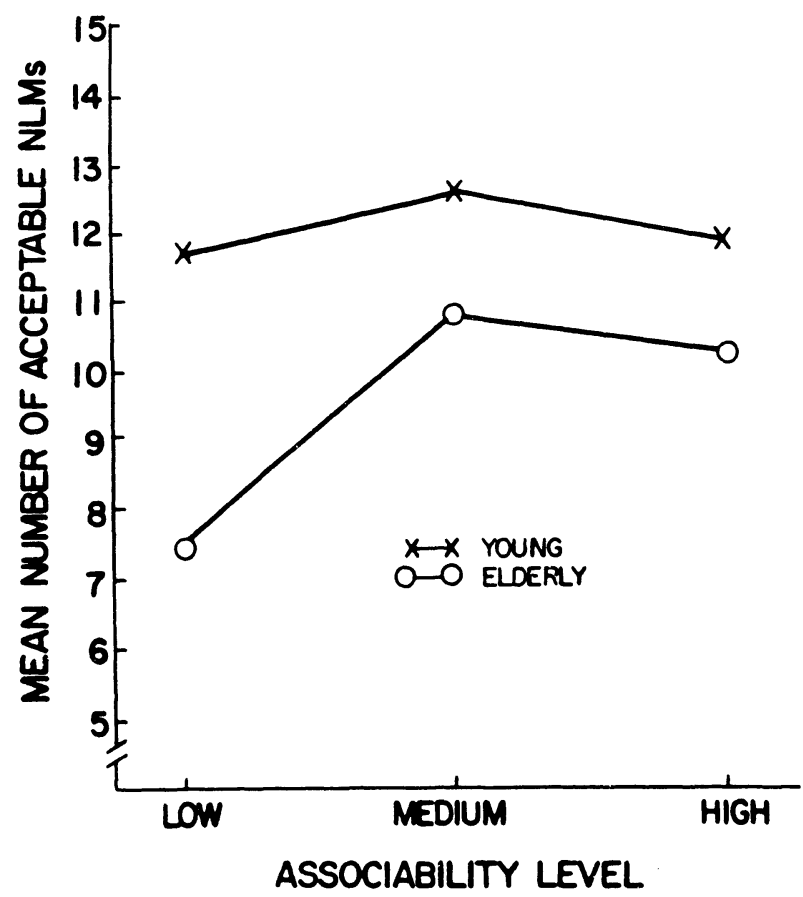

Figure 1. Percentage of two-word natural language mediators as a function of age and associability.

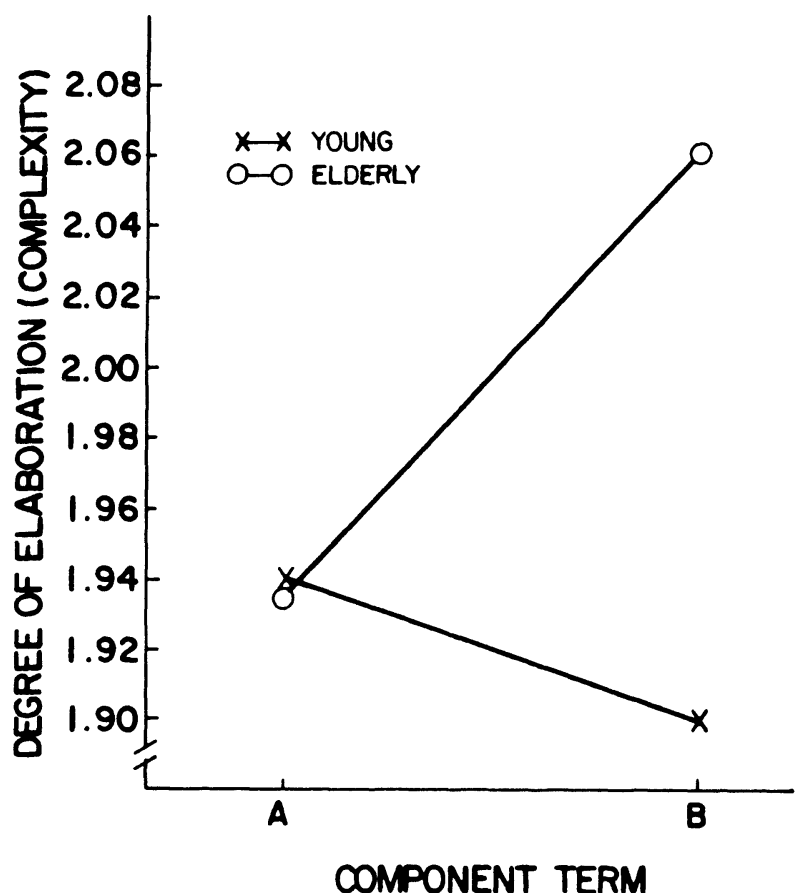

Figure 2. Complexity of natural language mediator components as a function of age.

items at both medium (mean $=1.84, S D=.34)$ and high (mean $=1.69, S D=.37$ ) associability levels. The latter two did not differ from each other $(p>.05)$.

Additionally, a significant associability $\times$ component term interaction $[F(2,68)=3.67, p<.05]$ found that B terms were embellished more than A terms only at the low associability level (Newman-Keuls).

A significant age $\times$ component term interaction $[F(1,34)=4.20, p<.05]$ is shown in Figure 2. For this interaction, a Newman-Keuls analysis indicated that older subjects embellished the B term more than the A term. The younger subjects did not differ significantly in their embellishment of these terms, although there was a trend toward greater A term embellishment. No other effects of interactions were significant at $p<.05$.

\section{DISCUSSION}

Montague and Kiess (1968) suggested that low-associability pairs should require more rules for generating NLMs and should therefore result in less efficient mediators. In the present study both young and elderly subjects generated more complex NLMs for low-associability pairs. However, overall NLM complexity cannot explain the observed differences in the mediational strategies of young and elderly subjects, since no such main effect was found in the present data or in the previous analysis conducted by Marshall et al. (1978).

There were age differences in the number of acceptable two-word mediators generated by the two groups. The associability factor was again important, since the outcome of fewer acceptable NLMs provided by the elderly was particularly apparent for low-associability pairs.

A further analysis of the available two-word NLMs found that older subjects had embellished the B term significantly more. The younger subjects showed a nonsignificant trend toward greater embellishment of the A term, and that coincides with earlier data reported by Owens et al. (1974) for their comparable young subjects. Earlier retention studies 
that reported asymmetry in the availability of $\mathrm{A}$ and $\mathrm{B}$ term components (usually showing greater availability, or recall, of the B term) for older adults (Kausler \& Lair, 1965; Winn \& J. W. Elias, 1978) did not control learning strategy; thus, whether such recall asymmetry pertains to rote, mediated, or both types of learned items is unknown.

No claims are made from the present data that two-word mediators produced from a single presentation of a CVC pair are, per se, superior to any other form of mediator. The paradigm manipulation was chosen only to allow the study of a particular function of mediators, the linking of the two components. The present data do suggest that there are factors of component term embellishment that may lead to further understanding of age differences in mediator formation and usage, and hence in memory.

\section{REFERENCES}

Burke, D. M., \& Light, L. L. (1981). Memory and aging: The role of retrieval processes. Psychological Bulletin, 90, 513-546.

Euias, M. F., Elias, P. K. , \& Elias, J. W. (1977). Adult developmental psychology. St. Louis: Mosby.

KAUSLER, D. H. (1982). Experimental psychology and human aging. New York: Wiley.
KAUSLER, D. H., \& LAIR, C. V. (1965). R-S ("backward”) pairedassociate learning in elderly subjects. Journal of Gerontology, 20, 29-31.

Marshall, P. H., Elias, J. W., Webber, S. M., Gist, B. A., WinN, F. J., Moore, S. A., \& KING, P. (1978). Age differences in verbal mediation: A structural and functional analysis. Experimental Aging Research, 4, 175-193.

Montague, W. E., \& Kiess, H. O. (1968). The associability of CVC pairs. Journal of Experimental Psychology Monographs, 78(2, Pt. 2).

OWens, J. M., Werder, P. R., \& Marshall, P. H. (1974). A component analysis of natural language mediators obtained in pairedassociate learning. Bulletin of the Psychonomic Society, 4, 512-514.

Prytulak, L. S. (1971). Natural language mediation. Cognitive Psychology, 2, 1-56.

WinN, F. J., \& Elias, J. W. (1978). Associative symmetry and item availability: Evidence for qualitative age differences in acquisition strategies. Experimental Aging Research, 4, 411-420.

(Manuscript received for publication June 23, 1986.) 